\title{
Speed of sound in porous materials
}

\author{
N RAMAKRISHNAN \\ Defence Metallurgical Research Laboratory, Kanchanbagh, Hyderabad 500258 , India \\ MS received 25 April 1994; revised 25 July 1994
}

\begin{abstract}
A set of analytical expressions which can be used for estimating the effective speed of sound in porous materials is presented along with a brief description of the micromechanical origins of the analytical equations. These equations are validated and the accuracies are compared using published experimental data corresponding to four different ceramic materials.
\end{abstract}

Keywords. Speed of sound; porous materials.

\section{Introduction}

Porous materials are commonly used in many engineering applications involving dynamic loading conditions. One of the important parameters which characterizes the dynamic response of a material is the speed of sound wave in the material medium. This investigation deals with the theoretical determination of the speed of sound in porous solids.

For an isotropic and homogeneous material, the speeds of longitudinal and transverse waves are given as (Schreiber et al 1973),

$$
\begin{aligned}
& \left(C_{1}\right)^{2}=\left\{K_{0}+(4 / 3) G_{0}\right\} / \rho, \\
& \left(C_{t}\right)^{2}=G_{0} / \rho,
\end{aligned}
$$

where $K_{0}$ and $G_{0}$ are bulk and shear moduli of the material, and $\rho$ denotes the density. Equations (1) and (2) can be adapted for porous materials also by simply replacing the moduli and the density by the effective moduli and the average density of the porous solid, provided the following conditions are satisfied (Christensen 1979):

(i) The porous solid is statistically isotropic and homogeneous where the pores are spatially distributed and oriented in a random fashion.

(ii) The wavelength of the characteristic sonic response of the material is reasonably larger than the average dimension of the pores.

Under these conditions, the equations representing the effective longitudinal and transverse sound speeds of the porous materials can be written in the form

$$
\begin{aligned}
& \left(C_{1}^{*}\right)^{2}=\left\{K^{*}+(4 / 3) G^{*}\right\} / \rho^{*}, \\
& \left(C_{1}^{*}\right)^{2}=G^{*} / \rho^{*},
\end{aligned}
$$

where the superscript * represents the effective properties. The average density is, by rule of mixtures,

$$
\rho^{*}=\rho(1-\theta),
$$

where $\theta$ is the porosity. But $K^{*}$ and $G^{*}$ do not obey the rule of mixtures and these 
are determined by various methods of micromechanics of composite materials. In this paper, we compare the speed of sound computed using different methods to the experimentally determined ones and present an analysis of the comparison.

\section{Effective sound speed}

Many empirical and theoretical equations were proposed in the past to express the effective moduli in terms of porosity and zero-porosity moduli. The empirical equations, because of the adjustable constants incorporated in them, are accurate in specific cases, but lack generality and physical insight. A detailed survey of the empirical equations and their relative merits has been presented by Dean and Lopez (1983). In the present investigation, we restrict our concern only to the theoretical estimation of the effective sound speed.

In most of the studies, the effective moduli of a porous solid are established, treating it as an extreme case of a two-phase composite. A number of methods (Christensen 1990), based on the micromechanics of multiphase composites, were introduced by various investigators to establish the effective moduli. These methods can be broadly classified under the following generic techniques: (a) composite sphere method (CSM), (b) self-consistent method (SCM) and (c) differential method (DM).

In CSM (Hashin 1962; Hashin and Shtrikman 1963), the two-phase geometry is simplified as an assembly of a set of composite spheres of different sizes, but maintaining the same volume fraction of the concentrically located spherical second phase in each composite sphere. Using this model, Hashin and Shtrikman (1963) established an effective bulk modulus and a set of bounds for the effective shear modulus. In one of our earlier studies (Ramakrishnan and Arunachalam 1990,1993), we proposed a modification of the CSM for porous solids in order to incorporate the effect of interaction between the pores. This resulted in an additional porositydependent factor in Hashin's expression for the effective bulk modulus. Moreover, we established a unique expression for the effective shear modulus satisfying Hashin's bounds, postulating a particular type of variation of the effective Poisson's ratio with porosity. In this case, the effective modulus $\left(M^{*}\right)$ is expressed in terms of the zeroporosity modulus $\left(M_{0}\right)$, the porosity $(\theta)$ and the zero-porosity Poisson's ratio $\left(v_{0}\right)$ as

$$
M^{*} / M_{0}=(1-\theta)^{2} /\left(1+b_{\mathrm{M}} \theta\right)
$$

where $b_{K}=\left(1+v_{0}\right) /\left\{2\left(1-2 v_{0}\right)\right\}, \quad b_{G}=\left(11-19 v_{0}\right) /\left\{4\left(1+v_{0}\right)\right\}$, and $b_{E}=2-3 v_{0}$. $M$ can be bulk, shear or Young's modulus and can be determined using the appropriate $b_{M}\left(b_{K}\right.$ for bulk, $b_{G}$ for shear and $b_{E}$ for Young's modulus). Substituting (6) in (3) and (4), the effective wave speeds $\left(C_{1}^{*}\right.$ and $\left.C_{t}^{*}\right)$ can be derived as

$$
\begin{aligned}
& \left(C_{1}^{*} / C_{1}\right)^{2}=\{(4+5 \theta)(1-\theta)\} /\left\{4\left(1+b_{K} \theta\right)\left(1+b_{G} \theta\right)\right\} \\
& \left(C_{t}^{*} / C_{t}\right)^{2}=(1-\theta) /\left(1+b_{G} \theta\right) .
\end{aligned}
$$

Subscripts 1 and $t$ denote longitudinal and transverse speeds respectively; $C_{1}$ and $C_{\mathrm{t}}$ are zero-porosity properties.

In SCM, the two-phase material is treated as a hypothetically homogeneous medium of unknown effective properties and then the stress field in a spherical or ellipsoidal inclusion in that medium is determined. Using the stress distribution, the expressions for the effective moduli are established averaging the stress field in the 
entire medium. Fröhlick and Sack (1946), using a type of self-consistent method, explained the viscoelastic properties of dispersions and subsequently Oldroyd (1955) and Rajagopal (1960) used it to determine the compressibility of general two-phase systems. Rajagopal (1961) extended the study to determine the sound velocity as well. Later, along similar lines, both Hill (1965) and Budiansky (1965) independently arrived at identical sets of equations for the effective moduli. Extended to porous material, these equations can be expressed as (with the same notations as earlier)

where

$$
\left(G^{*} / G_{0}\right)^{2}+p_{1}\left(G^{*} / G_{0}\right)+q_{1}=0,
$$

and

$$
\begin{aligned}
& p_{1}=\left\{(1 / 4)\left(1+v_{0}\right)(3-\theta) /\left(1-2 v_{0}\right)\right\}+\{(5 \theta-2) / 2\} \\
& q_{1}=(3 / 4)\left(1+v_{0}\right)(2 \theta-1) /\left(1-2 v_{0}\right)
\end{aligned}
$$

$$
\left(K^{*} / K_{0}\right)=(1-\theta)^{2} /\left\{1+\left(G_{0} / G^{*}\right) b_{K} \theta\right\}
$$

$b_{K}$ is defined in (6). The effective wave speeds, $C_{1}^{*}$ and $C_{t}^{*}$, can be determined combining (3) to (5) and (9) and (10). In this case the expressions for effective moduli are not in an explicit form and therefore it may be more convenient to leave them as a set of equations.

In differential method also, the two-phase material is assumed to be a homogeneous medium and the differentials of the effective moduli are established using the stress field around an infinitesimal spherical or ellipsoidal inclusion in that medium. Recently Zimmerman (1991) employed this method and established the effective moduli, which can be expressed as

where

$$
G^{*} / G_{0}=(1-\theta)^{2}\left\{p_{2}+q_{2}\left(G^{*} / G_{0}\right)^{3 / 5}\right\}^{1 / 3}
$$

where

$$
\begin{aligned}
& p_{2}=(2 / 3)\left(1+v_{0}\right) /\left(1-v_{0}\right), \\
& q_{2}=(1 / 3)\left(1-5 v_{0}\right) /\left(1-v_{0}\right), \\
& K^{*} / K_{0}=\left\{\left(G^{*} / G_{0}\right) / b_{K}\right\}\left\{1 /\left(1+p_{3}\right)\right\},
\end{aligned}
$$

and

$$
b_{K}=\left(1+v_{0}\right) /\left\{2\left(1-2 v_{0}\right)\right\}
$$

$$
p_{3}=(1-5 v)\left(G^{*} / G_{0}\right)^{3 / 5} /\left(1+v_{0}\right) \text {. }
$$

The effective moduli determined using (11) and (12) can be substituted in (3) and (4) to obtain the effective sound speeds. As (11) and (12) are not in an explicit form, $C_{1}^{*}$ and $C_{t}^{*}$ also cannot be expressed in any explicit form.

\section{Discussion}

In this section we present a comparative picture of the applicability and the accuracy of the various theoretical equations in predicting the experimentally measured effective sound speeds. For this purpose, we chose four sets of published experimental data (Janowski and Rossi 1967; Manning et al 1969; Hunter et al 1974; Hunter and Graddy 1976) on the effective Young's and shear moduli of $\mathrm{MgO}\left(v_{0}=0.174\right), \mathrm{Lu}_{2} \mathrm{O}_{3}\left(v_{0}=0.273\right)$, $\mathrm{Ho}_{2} \mathrm{O}_{3}\left(v_{0}=0.290\right)$ and $\mathrm{Sm}_{2} \mathrm{O}_{3}\left(v_{0}=325\right)$. The sets of data were gathered by a 

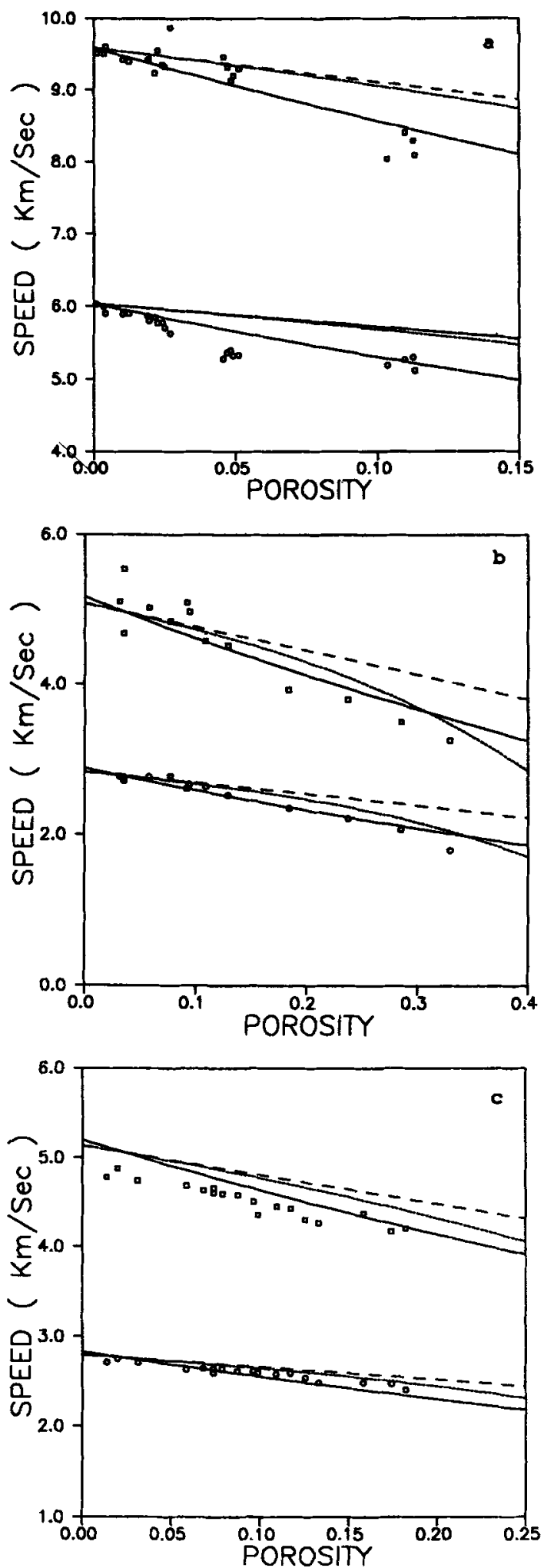

Figure 1. a-c. 


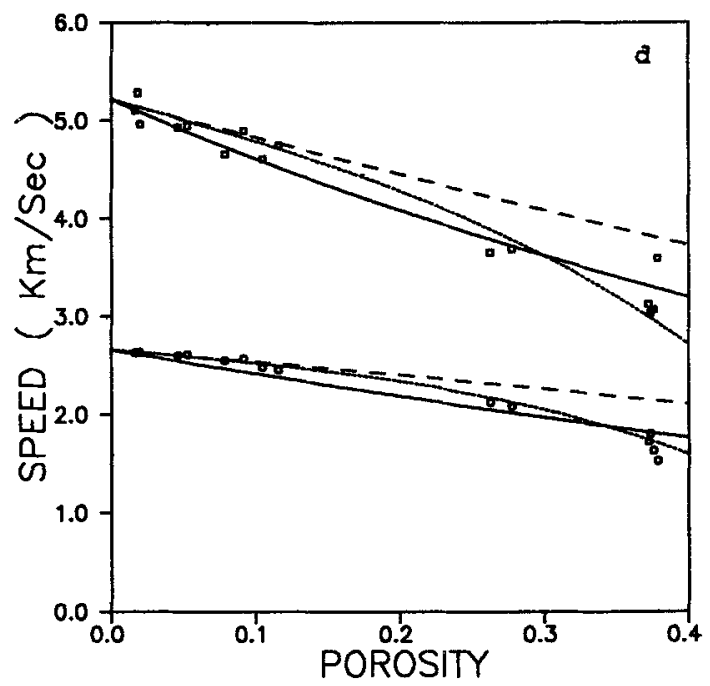

Figure 1. Variation of effective sound speed with porosity: $(\square)$ speed of longitudinal wave; (O) speed of transverse wave; (-) Ramakrishnan and Arunachalam (1990, 1993), CSM; (..) Hill (1965) and Budiansky (1965), SCM; (--) Zimmerman (1991), DM. (a) MgO( $\left.v_{0}=0.172\right)$ (Janowsky and Rossi 1967), (b) $\mathrm{Lu}_{2} \mathrm{O}_{3}\left(v_{0}=0.274\right.$ )(Hunter and Graddy 1976), (c) $\mathrm{Ho}_{2} \mathrm{O}_{3}$ $\left(v_{0}=0.290\right)$ (Manning et al 1969), and (d) $\mathrm{Sm}_{2} \mathrm{O}_{3}\left(v_{0}=0.325\right)$ (Hunter et al 1974).

digitization process and compiled into a common format. The effective sound speeds were computed using (3) and (4). The comparison of the equations is presented along with the experimental data in figures 1 (a) to (d), sequenced in the increasing order of the zero-porosity Poisson's ratio. The scatter in the effective longitudinal speed is more than that of the effective transverse speed. This is because of the sensitiveness of the bulk modulus to the error in the zero-porosity material properties and only the longitudinal speed depends explicitly on the bulk modulus.

In the case of $\mathrm{MgO}$ (figure 1a), for which the zero-porosity Poisson's ratio is 0-172, both Hill-Budiansky's and Zimmerman's equations overestimate the effective speeds whereas the equations of Ramakrishnan and Arunachalam have a relatively better agreement with the experimental data. With increase of $v_{0}$ (figures $1 b-d$ ), the accuracy of Hill-Budiansky's equations improves. Ramakrishnan-Arunachalam's equations behave satisfactorily in this range as well, except that they mildly overestimate the effective speeds in the intermediate porosity range of about $30-40 \%$. Invariably in all the cases, Zimmerman's model overestimates the effective speeds; nevertheless, the accuracy improves with increase in $v_{0}$. A quantitative assessment of the accuracy of these equations becomes difficult as the accuracy strongly depends on the zeroporosity Poisson's ratio and the porosity, and in a few cases the scatter in the experimental data is of the order of the accuracy itself. Nevertheless, figures 1(a) - (d) conclusively demonstrate that a reasonably accurate prediction is possible with the theoretical equations considered in this investigation.

\section{References}


Christensen R M 1990 J. Mech. Phys. Solids 38379

Dean E A and Lopez J A $1983 \mathrm{~J}$. Am. Ceram. Soc. 66366

Fröhlich H and Sack R 1946 Proc. R. Soc. (London) A185 415

Hashin Z 1962 J. Appl. Mech. 29143

Hashin Z and Shtrikman S 1963 J. Mech. Phys. Solids 11127

Hill R 1965 J. Mech. Phys. Solids 13213

Hunter Jr. O, Korklan H J and Suchomel R R 1974 J. Am. Ceram. Soc.-Diss. \& Notes 57267

Hunter Jr. O and Graddy Jr. G E $1976 \mathrm{~J}$. Am. Ceram. Soc.-Diss. \& Notes 5982

Janowski K R and Rossi R C 1967 J. Am. Ceram. Soc. 50599

Manning W R, Hunter Jr. O and Powell Jr. B R 1969 J. Am. Ceram. Soc. 52436

Oldroyd J G 1955 Proc. R. Soc. (London) A232 567

Rajagopal E S 1960 Z. Phys. Chem. N. F. 23342

Rajagopal E S 1961 Acustica 11178

Ramakrishnan N and Arunachalam V S 1990 J. Mater. Sci. 253930

Ramakrishnan N and Arunachalam V S $1993 \mathrm{~J}$. Am. Ceram. Soc. 762745

Schreiber E, Anderson O L and Soga N 1973 Elastic constants and their measurement (McGraw-Hill Book Co.)

Zimmerman R W 1991 Mech. Mater. 1217 